\title{
Pitfalls of Using PQ-Trees in Automatic Graph Drawing ${ }^{\star}$
}

\author{
Michael Jünger ${ }^{1}$, Sebastian Leipert ${ }^{2}$ and Petra Mutzel ${ }^{3}$ \\ 1 Institut für Informatik, Universität zu Köln, mjuenger@informatik.uni-koeln.de \\ ${ }^{2}$ Institut für Informatik, Universität zu Köln, leipert@informatik.uni-koeln.de \\ ${ }^{3}$ Max-Planck-Institut für Informatik, Saarbrücken, mutzel@mpi-sb.mpg.de
}

\begin{abstract}
A number of erroneous attempts involving $P Q$-trees in the context of automatic graph drawing algorithms have been presented in the literature in recent years. In order to prevent future research from constructing algorithms with similar errors we point out some of the major mistakes.

In particular, we examine erroneous usage of the $P Q$-tree data structure in algorithms for computing maximal planar subgraphs and an algorithm for testing leveled planarity of leveled directed acyclic graphs with several sources and sinks.
\end{abstract}

\section{Introduction}

A $P Q$-tree is a powerful data structure that represents the permutations of a finite set in which the members of specified subsets occur consecutively, and in which updates require linear time. This data structure has been introduced by Booth and Lueker (1976) to solve the problem of testing for the consecutive ones property. The most well known applications of $P Q$-trees in automatic graph drawing are planarity testing (see Lempel et al. (1967), Booth and Lueker (1976)) and embedding (see Chiba et al. (1985)). Therefore $P Q$-trees have become standard tools in automatic graph drawing systems.

Other attempts to use algorithms based on $P Q$-trees for automatic graph drawing problems have not been successful. One well known example is the computation of maximal planar subgraphs. Given a simple, connected graph $G=(V, E)$ with $n$ vertices and $m$ edges, a planar subgraph $G^{\prime}$ of $G$ is a maximal planar subgraph, if for all edges $e \in G-G^{\prime}$ the addition of $e$ to $G^{\prime}$ destroys planarity. Several efforts have been made in the literature to solve the problem with $P Q$-trees following a certain strategy that was first presented by Ozawa and Takahashi (1981). They describe an $O(n m)$ algorithm using $P Q$ tree techniques based on the vertex addition algorithm of Lempel et al. (1967). Jayakumar et al. (1986) show that in general this algorithm does not determine a maximal planar subgraph. Moreover, the resulting planar subgraph may not

\footnotetext{
* Partially supported by DFG-Grant Ju204/7-2, Forschungsschwerpunkt "Effiziente Algorithmen für diskrete Probleme und ihre Anwendungen" and ESPRIT Long Term Research Project Nr. 20244 (ALCOM-IT)
} 
even contain all vertices. Jayakumar et al. (1989) present an algorithm called PLANARIZE that computes a spanning planar subgraph $G_{p}$ of $G$ in $\mathrm{O}\left(n^{2}\right)$ time. Furthermore, they present an algorithm called MAX-PLANARIZE that augments $G_{p}$ to a subgraph $G^{\prime}$ of $G$ by adding additional edges in $O\left(n^{2}\right)$ time. They claim that $G^{\prime}$ is a maximal planar subgraph of $G$ if $G_{p}$ (the result of phase 1 of the two phase algorithm) turns out to be biconnected. Kant (1992) shows that this algorithm is incorrect, and suggests a modification of the second phase of the algorithm that augments $G_{p}$ to a maximal planar subgraph of $G$, even if $G_{p}$ is not biconnected, maintaining $O\left(n^{2}\right)$ time requirement. In Jünger et al. (1996) we show that this modification is not correct either. Here we point out a substantial flaw in both the original and the modified two phase algorithm that was not detected previously. This is the subject of section 2 .

$P Q$-trees have also been proposed by Heath and Pemmaraju (1996a, 1996b) to test leveled planarity of leveled directed acyclic graphs with several sources and sinks. In section 3 we show why this application leads to an incorrect algorithm as well. Since this "algorithm" is the only attempt to prove the polynomial time complexity in the literature, the complexity status of leveled planarity testing is still open. Only in the special case in which there is only one source (or only one sink) the algorithm is correct and implies linear time solvability, but this has already been shown previously by Di Battista and Nardelli (1988).

\section{Case study: maximal planar subgraphs}

\section{1 $P Q$-trees for planarity testing}

Let $G=(V, E)$ be a simple graph with $n$ vertices and $m$ edges. A graph is planar, if it can be embedded in the plane without any edge crossings. A graph is obviously planar, if and only if its biconnected components are planar. We therefore assume that $G$ is biconnected. The planarity testing algorithm of Lempel et al. (1967) first labels the vertices of $G$ as $1,2 \ldots, n$ using an stnumbering (see Even and Tarjan (1976)). A numbering of the vertices of $G$ by $1,2 \ldots, n$ is an st-numbering, if the vertices " 1 " and " $n$ " are adjacent and each other vertex $j$ is adjacent to two vertices $i$ and $k$ such that $i<j<k$. The vertex 1 is denoted by $s$ and the vertex $n$ is denoted by $t$. The $s t$-numbering induces an orientation of the graph, in which every edge is directed from the incident vertex with the higher st-number towards the incident vertex with the lower $s t$-number. From now on we refer to the vertices of $G$ by their st-numbers and call an edge $(u, v)$, with $v<u$, incoming edge of $v$ and outgoing edge of $u$.

For $1 \leq k \leq n$, let $G_{k}$ denote the subgraph of $G$ induced by the vertex set $V_{k}:=\{1,2, \ldots, k\}$. The graph $G_{k}^{\prime}$ arises from $G_{k}$ as follows: For each edge $e=(u, v)$, where $v \in V_{k}$ and $u \in V \backslash V_{k}$, we introduce a virtual vertex $u_{e}$ with label $u$ and a virtual edge $\left(u_{e}, v\right)$. Let $B_{k}$ be a planar embedding of $G_{k}^{\prime}$ such that all virtual vertices are placed on the outer face. Then, $B_{k}$ is called a bush form. It has been shown by Lempel et al. (1967) that $G$ is planar, if and only if for every $B_{k}, k=1,2, \ldots, n-1$, there exists a bush form $B_{k}^{\prime}$ isomorphic to $B_{k}$, such that all virtual vertices in $B_{k}^{\prime}$ labeled $k+1$ appear consecutively. 
The $P Q$-tree $T_{k}$ corresponding to the bush form $B_{k}$ is a rooted ordered tree that consists of three types of nodes:

1. Leaves in $T_{k}$ represent virtual edges in $B_{k}$.

2. $P$-nodes in $T_{k}$ represent cutvertices in $B_{k}$.

3. Q-nodes represent maximal biconnected components in $B_{k}$.

The frontier of a $P Q$-tree is the sequence of all leaves of $T_{k}$ read from left to right. The frontier of a node $X$, denoted by frontier $(X)$, is the sequence of its descendant leaves read from left to right.

Let $E_{k+1}$ denote the set of leaves in $T_{k}$ that correspond to the virtual vertices labeled $k+1$. A node $X$ is called full, if all leaves in its frontier are in $E_{k+1}$. A node $X$ is empty, if its frontier does not contain any leaf of $E_{k+1}$. Otherwise, $X$ is called partial. A node is called pertinent, if it is full or partial. The pertinent subtree is the smallest connected subtree that contains all leaves of $E_{k+1}$ in its frontier. The root of the pertinent subtree is called pertinent root. Two $P Q$ trees are equivalent, if one can be obtained from the other by one or more of the following operations:

1. Permuting the children of a $P$-node.

2. Reversing the order of the children of a $Q$-node.

These operations are called equivalence transformations and describe equivalence classes on the set of all $P Q$-trees. An equivalence class of $P Q$-trees corresponds to a class of permutations called the permissible permutations.

It has been shown by Booth and Lueker (1976) that $B_{k}^{\prime}$ exists if and only if $T_{k}$ can be converted into an equivalent $P Q$-tree $T_{k}^{\prime}$ such that all pertinent leaves appear consecutively in the frontier of $T_{k}^{t}$. Booth and Lueker (1976) have defined a set of patterns and replacements called templates that can be used to reduce the $P Q$-tree such that the leaves corresponding to edges of the set $E_{k+1}$ appear consecutively in all permissible permutations. To construct $T_{k+1}$ from $T_{k}$ they first reduce $T_{k}$ by use of the templates and then replace all leaves corresponding to virtual edges incident to vertices labeled $k+1$ by a $P$-node, whose children are the leaves corresponding to the incoming edges of the vertex $k+1$ in $G$.

The planarity testing algorithm now starts with $T_{1}$ and constructs a sequence of $P Q$-trees $T_{1}, T_{2}, \ldots$ If the graph is planar, the algorithm terminates after constructing $T_{n-1}$. Otherwise it terminates after detecting the impossibility of reducing some $T_{k}, 1 \leq k<n$.

\subsection{Principle of an approach for planarization}

The basic idea of a planarization algorithm using $P Q$-trees presented by Jayakumar et al. (1989) is to construct the sequence of $P Q$-trees $T_{1}, T_{2}, \ldots, T_{n-1}$ by deleting an appropriate number of pertinent leaves every time the reduction fails, such that the resulting $P Q$-tree becomes reducible. In every step of the algorithm PLANARIZE, a maximal consecutive sequence of pertinent leaves is computed by using a $[w, h, a]$-numbering (see Jayakumar et al. (1989)). All pertinent leaves 
that are not adjacent to the maximal pertinent sequence are removed from the $P Q$-tree in order to make it reducible. Hence the edges corresponding to the leaves are removed from $G$ and the resulting graph $G_{p}$ is planar.

It has been shown by Jayakumar et al. (1989) that the graph $G_{p}$ computed by PLANARIZE is not necessarily maximal planar. The authors therefore suggest to apply a second phase MAX-PLANARIZE, also based on $P Q$-trees. Knowing which edges have been removed from $G$ to construct $G_{p}$, edges from $G-G_{p}$ are added back to $G_{p}$ in the second phase without destroying planarity.

During the reduction of a vertex $v$, there may exist nonpertinent leaves that are in all permissible permutations of the $P Q$-tree $T_{v-1}$ between a pertinent leaf $l_{v}$ and its maximal pertinent sequence. This maximal pertinent sequence has been determined with the help of the $[w, h, a]$-numbering. In order to make the tree $T_{v-1}$ reducible, the leaf $l_{v}$ is removed from the tree and the corresponding edge is removed from the graph $G$, guaranteeing that the subgraph $G_{p}$ will be planar. However, it may occur that the nonpertinent leaves that are positioned between $l_{v}$ and its maximal pertinent sequence in $T_{v-1}$, are removed as well from a tree $T_{k}, v \leq k<n$, in order to obtain reducibility. Therefore, there is no need to remove the edge corresponding to $l_{v}$ from the graph $G$.

In order to find leaves such as $l_{v}$, Jayakumar et al. (1989) use the algorithm MAX-PLANARIZE. In step $i$, both PLANARIZE as well as MAX-PLANARIZE reduce the same vertex $i$. The difference between the $P Q$-trees in the two algorithms is, according to the authors, that all leaves that have been deleted in PLANARIZE are ignored in MAX-PLANARIZE from the moment they are introduced into the tree until they get pertinent. This causes the nonpertinent leaves between the pertinent leaf $l_{v}$ and its maximal pertinent sequence to be ignored. Hence $l_{v}$ is adjacent to its maximal pertinent sequence and the corresponding edge can be added back to $G_{p}$, while the leaves between $l_{v}$ and the maximal pertinent sequence are removed from the $P Q$-tree.

\subsection{On the incorrectness of the algorithm}

While some incorrect details of the approach of Jayakumar et al. have been described in a technical report by Kant (1992), who attempted to correct the algorithm, a major problem has not been detected.

Jayakumar et al. assume that the maximal planar subgraph $G_{p}$ is biconnected for the correct application of the Lempel-Even-Cederbaum algorithm. Furthermore, as they have stated correctly, this is necessary in order to have an $s t$-numbering. Nevertheless, the $P Q$-trees in MAX-PLANARIZE are constructed according to the $s t$-numbering that was computed for the graph $G$.

As a matter of fact, the st-numbering of $G$ does not imply an st-numbering of any subgraph $G_{p}$ even if the subgraph $G_{p}$ is biconnected. This results in two problems, of which one is crucial and cannot be dealt with even by the ideas described by Kant (1992).

Both problems are based on the fact that during the application of PLANARIZE for some vertices of $V$ all incoming edges may be deleted from the 
graph while the resulting graph $G_{p}$ stays biconnected. In this abstract, we consider only the crucial problem. The other problem is described in detail by Jünger et al. (1996).

The planarization algorithm of Jayakumar et al. (1989) does not obey an important invariant implied by the following lemma, shown by Even (1979).

Lemma 2.1 Let $G=(V, E)$ be a planar graph with an st-numbering and let $1 \leq k \leq n$. If the edge $(t, s)$ is drawn on the boundary of the outer face in an embedding of $G$, then all vertices and edges of $G-G_{k}$ are drawn in the outer face of the plane subgraph $G_{k}$ of $G$.

This result allowed Lempel et al. (1967) to transform the problem of planarity testing to the construction of a sequence of bush forms $B_{k}, 1 \leq k \leq n$. For a planar graph $G$, edges and vertices that have not been introduced into the current subgraph $G_{k}$ are always embedded into the outer face of $G_{k}$.

The approach of Jayakumar et al. (1989) does not obey this invariant in the second phase. There exist edges that have to be embedded into an inner face of some $G_{k}$, even if $(t, s)$ is drawn on the outer face. Due to the above lemma, the correction step MAX-PLANARIZE only considers edges for reintroduction into the planar subgraph $G_{p}$ that are on the outer face of the current graph $G_{k}$. Since the numbering that is used to determine the order in which the vertices are reduced does not correspond to an $s t$-numbering of $G_{p}$ in general, the algorithm of Jayakumar et al. (1989) ignores edges that have to be added into an inner face of the embedding of a current graph $G_{k}$. This fact is fatal, as we are about to show now.

In Fig. 1, a part of a bush form $B_{k-1}, 1<k \leq n$ of a graph $G$ is shown. The virtual vertices corresponding to the vertex $k$ are labeled $k_{1}, k_{2}, \ldots, k_{5}$ and all other virtual vertices are left unlabeled. The corresponding part of the $P Q$-tree is shown in Fig. 2. Obviously, there do not exist any reversions or permutations such that the virtual vertices of $k$ occupy consecutive positions. Hence, the graph $G$ is not planar. Applying the $[w, h, a]$-numbering of Jayakumar et al. (1989) allows us to delete the virtual vertex $k_{5}$ and to reduce the other four vertices $k_{1}, k_{2}, k_{3}, k_{4}$. The resulting bush form $B_{k}$ is planar and the relevant part is shown in Fig. 3. Figure 4 shows the corresponding part of the $P Q$-tree. Assume now that all descendants of $k$ have to be removed from the $P Q$-tree in a later step. Hence all incoming edges incident on $k$ are removed from the tree. Now assume further that there exists a path $v_{1}, v_{2}, \ldots, v_{l}$ in $G_{p}$ such that

- for all $i, j, 1 \leq i<j \leq l$ the inequality $v_{i}<v_{j}$ holds,

- the edge $\left(v_{2}, v_{1}\right)$ corresponds to one of the virtual edges that are between the leaf $k_{5}$ and the maximal pertinent sequence $k_{1}, k_{2}, k_{3}, k_{4}$ in all $P Q$-trees equivalent to $T_{k-1}$,

$-v_{l}=t$.

This path guarantees that all outgoing edges of the vertex $k$ cannot be embedded into the outer face of the embedding of $B_{k-1}$ without crossing an edge on this path. Hence the edge $e_{k_{5}}$ corresponding to the leaf $k_{5}$ is not considered 


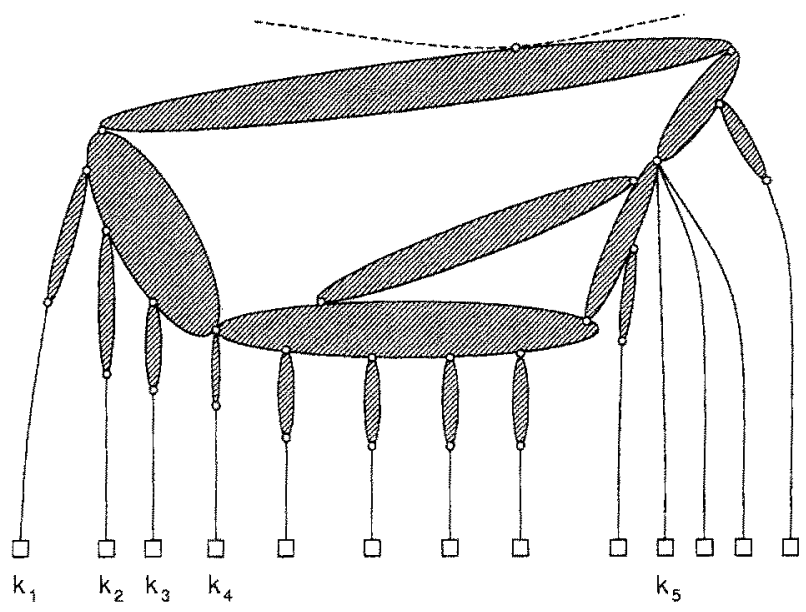

Fig. 1. Part of a bush form $B_{k-1}$

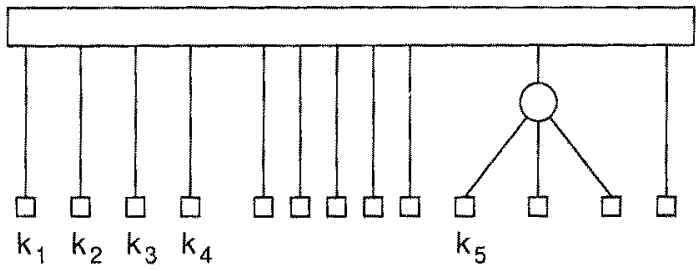

Fig. 2. Part of a $P Q$-tree corresponding to bush form $B_{k-1}$

by the algorithm MAX-PLANARIZE as being an edge that does not destroy planarity. Therefore, $e_{k_{5}}$ is not added back to the planar subgraph $G_{p}$.

Nevertheless adding the edge $e_{k_{5}}$ to $G_{p}$ may not destroy planarity of $G_{p}$ as is shown in our example in Fig. 5. Since all incoming edges of the vertex $k$ have been deleted by PLANARIZE and are not added back by MAX-PLANARIZE, it may be possible to swap the vertex $k$ into an inner face of the embedding of $B_{k}$ such that the virtual vertex $k_{5}$ can be identified with $k$ and the edge $e_{k_{5}}$ is embedded into the bush form $B_{k}$ without destroying planarity.

Therefore, the strategy of using $P Q$-trees presented by Jayakumar et al. (1989) does not compute a maximal planar subgraph in general. Furthermore, we point out that the same problem holds for the modified version of this algorithm, presented by Kant (1992). This version follows a similar strategy of computing a spanning planar subgraph $G_{p}$ using PLANARIZE and then adding edges that do not destroy planarity in a second phase. The order of reductions that is used to insert vertices into existing bush forms is the same as the one implied by the $s t$-numbering on $G$. Hence this approach is not able to compute a maximal planar subgraph for the same reason. 


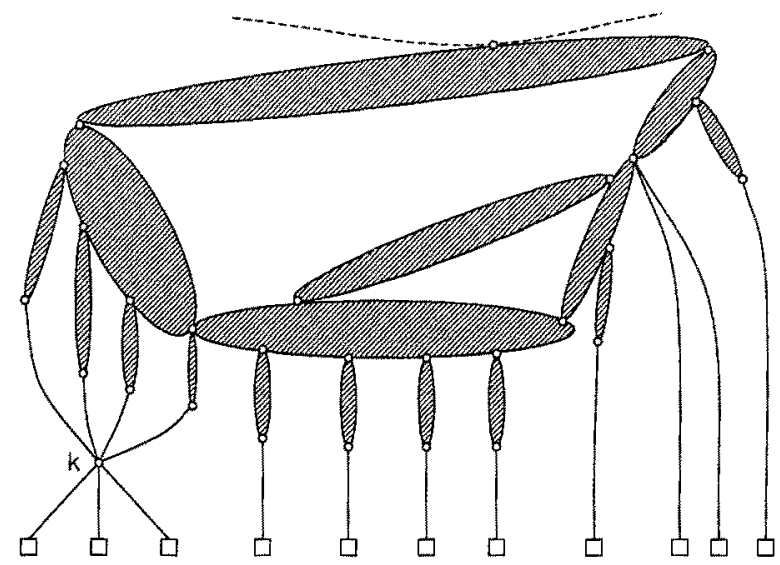

Fig. 3. Part of a bush form $B_{k}$

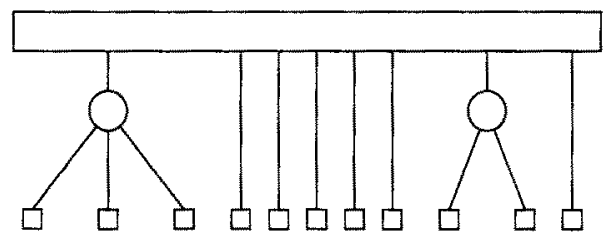

Fig. 4. Part of a $P Q$-tree corresponding to bush form $B_{k}$

Summarizing, we state the following lemma that has been shown in the discussion above.

Lemma 2.2 Let $G=(V, E)$ be a nonplanar graph. Let $G_{p}=\left(V, E_{p}\right), E_{p} \subseteq E$, be a planar subgraph of $G$, such that $G_{p}$ was obtained from $G$ by

1. computing an st-numbering for all vertices and

2. applying the algorithm of Lempel et al. (1967) constructing a sequence of bush forms $B_{k}, 1 \leq k \leq n$, by embedding a maximal number of outgoing edges of a vertex $k, 1<k \leq n$, in the outer face of $B_{k-1}$ without crossings, deleting all other outgoing edges of $k$.

Let $G_{p}^{\prime}=\left(V, E_{p}^{\prime}\right)$, be a planar subgraph of $G$ such that

1. $E_{p} \subseteq E_{p}^{\prime} \subseteq E$,

2. the graph $G_{p}^{\prime}$ is computed by constructing a sequence of bush forms $B_{k}^{\prime}$, $1 \leq k \leq n$, based on the st-numbering used for determining $G_{p}$, and possibly embedding outgoing edges $e \in E \backslash E_{p}$ of every vertex $k, 1<k \leq n$, without crossings in the outer face of $B_{k-1}$.

Then the subgraph $G_{p}^{\prime}$ is not necessarily maximal planar. 


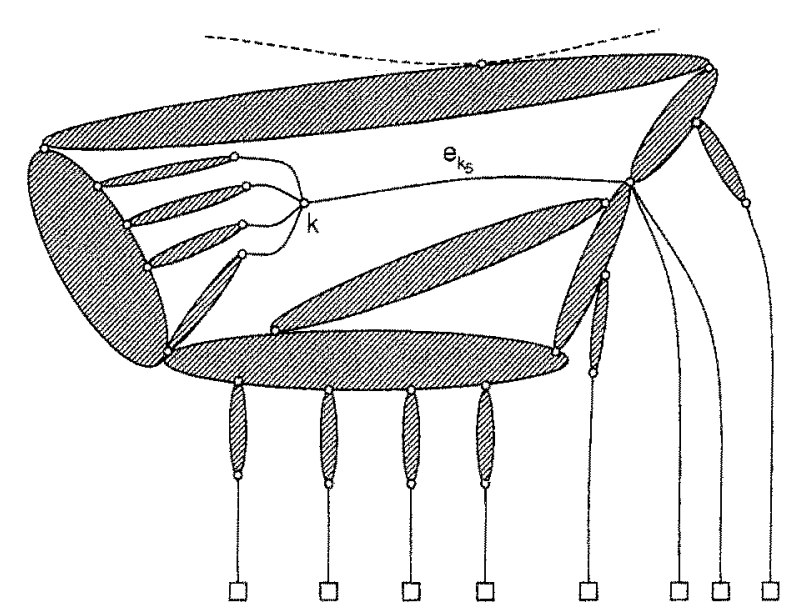

Fig. 5. Part of a bush form $B_{k}$ with $e_{k_{5}}$ embedded

Considering a computation of an st-numbering for the planar subgraph $G_{p}$ in order to augment $G_{p}$ to a maximal planar subgraph of $G$ and then construct a sequence of bush forms $B_{k}^{\prime}, 1 \leq k \leq n$, is aggravated by the fact that the graph $G_{p}$ is not biconnected in general. Furthermore, the difference between the bush forms of the first phase and the second phase may result in the deletion of the edges of $G_{p}$ as soon as edges of $E \backslash E_{p}$ are added to $G_{p}$.

\section{Case study: leveled planarity testing}

\subsection{Principle of an approach for recognizing leveled planar dags}

Let $G=(V, E)$ be a directed acyclic graph. A leveling of $G$ is a function lev : $V \rightarrow \mathbb{Z}$ mapping the nodes of $G$ to integers such that $\operatorname{lev}(v)=\operatorname{lev}(u)+1$ for all $(u, v) \in E . G$ is called a leveled dag if it has a leveling. If $l e v(v)=j$, then $v$ is a level- $j$ vertex. Let $V_{j}=l e v^{-1}(j)$ denote the set of level- $j$ vertices. Each $V_{j}$ is a level of $G$.

For the rest of this section, we consider $G$ to be a leveled dag with $m \in \mathbb{N}$ levels. An embedding of $G$ in the plane is called leveled if the vertices of every $V_{j}$, $1 \leq j \leq m$, are placed on a horizontal line $l_{j}=\{(x, m-j) \mid x \in \mathbb{R}\}$, and every edge $(u, v) \in E, u \in V_{j}, v \in V_{j+1}$ is drawn as straight line segment between the lines $l_{j}$ and $l_{j+1}$. A leveled embedding of $G$ is called leveled planar if no two edges cross except at common endpoints. A leveled dag is leveled planar, if it has a leveled planar embedding. The $\operatorname{dag} G$ is obviously leveled planar, if all its components are leveled planar. We therefore assume that $G$ is connected.

A leveled embedding of $G$ determines for every $V_{j}, 1 \leq j \leq m$, a total order $\leq_{j}$ of the vertices of $V_{j}$, given by the left to right order of the nodes on $l_{j}$. In order to test whether a leveled embedding of $G$ is leveled planar, it is sufficient 
to find an order of the vertices of every set $V_{j}, 1 \leq j<m$, such that for every pair of edges $\left(u_{1}, v_{1}\right),\left(u_{2}, v_{2}\right) \in E$ with $\operatorname{lev}\left(u_{1}\right)=\operatorname{lev}\left(u_{2}\right)=j$ and $u_{1} \leq_{j} u_{2}$ it follows that $v_{1} \leq_{j+1} v_{2}$. Apparently, the ordering $\leq_{j}, 1 \leq j \leq m$, describes a permutation of the vertices of $V_{j}$. Let $G_{j}$ denote the subgraph of $G$, induced by $V_{1} \cup V_{2} \cup \ldots \cup V_{j}$. Unlike $G, G_{j}$ is not necessarily connected.

The basic idea of the leveled planarity testing algorithm presented by Heath and Pemmaraju Heath and Pemmaraju (1996a, 1996b) is to perform a top-down sweep processing the levels in the order $V_{1}, V_{2}, \ldots, V_{m}$ computing for every level $V_{j}, 1 \leq j \leq m$, a set of permutations of the vertices of $V_{j}$ that appear in some leveled planar embedding of $G_{j}$. In case that the set of permutations for $G_{m}$ is not empty, the graph $G=G_{m}$ is obviously leveled planar.

As long as the graph $G_{j}$ is connected for some $j \in\{1,2,3, \ldots, m\}$ standard $P Q$-tree techniques similar to the ones used in the planarity test can be applied in order to determine the required set of permutations (see Di Battista and Nardelli (1988)). In case that $G_{j}, 1 \leq j<m$, consists of more than one connected component, Heath and Pemmaraju suggest to use a $P Q$-tree for every component and formulate a set of rules of how to merge components $F_{1}$ and $F_{2}$, respectively their corresponding $P Q$-trees $T_{1}$ and $T_{2}$, if $F_{1}$ and $F_{2}$ both are adjacent to some vertex $v \in V_{j+1}$.

The authors first reduce the pertinent leaves of $T_{1}$ and $T_{2}$ corresponding to the vertex $v$. After successfully performing the reduction, the consecutive sequence of pertinent leaves is replaced by a single pertinent representative in both $T_{1}$ and $T_{2}$. Going up one of the trees $T_{i}, i \in\{1,2\}$, from its pertinent representative, an appropriate position is searched, allowing the tree $T_{j}, j \neq i$ to be placed into $T_{i}$. After successfully performing this step the resulting tree $T^{\prime}$ has two pertinent leaves corresponding to the vertex $v$, which again are reduced. If any of the steps fails, Heath and Pemmaraju state that the graph $G$ is not leveled planar.

Merging two $P Q$-trees $T_{1}$ and $T_{2}$ corresponds to merging the two components $F_{1}$ and $F_{2}$ and is accomplished using certain informations that are stored at the nodes of the $P Q$-trees. For any subset $S$ of the set of vertices in $V_{j}, 1 \leq j \leq m$, that belong to a component $F$, define $\operatorname{ML}(S)$ to be the greatest $d \leq j$ such that $V_{d}, V_{d+1}, \ldots, V_{j}$ induces a dag in which all nodes of $S$ occur in the same connected component. For a $Q$-node $q$ in the corresponding $P Q$-tree $T_{F}$ with ordered children $r_{1}, r_{2}, \ldots, r_{t}$ maintain in node $q$ integers denoted $\mathrm{ML}\left(r_{i}, r_{i+1}\right)$, where $1 \leq i<t$, satisfying $\mathrm{ML}\left(r_{i}, r_{i+1}\right)=\mathrm{ML}\left(\right.$ frontier $\left(r_{i}\right) \cup$ frontier $\left.\left(r_{i+1}\right)\right)$. For a $P$-node $p$ maintain in $p$ a single integer denoted $\mathrm{ML}(p)$ that satisfies $\mathrm{ML}(p)=\mathrm{ML}($ frontier $(p))$. Furthermore define $\mathrm{LL}(F)$ to be the smallest $d$ such that $F$ contains a vertex in $V_{d}$ and maintain this integer at the root of the corresponding $P Q$-tree.

Using these LL- and ML-values, Heath and Pemmaraju (1996a, 1996b) describe a set of rules how to connect two $P Q$-trees claiming that the pertinent leaves of the new tree $T^{\prime}$ are reducible if and only if the corresponding component $F^{\prime}$ is leveled planar. 


\subsection{On the incorrectness of the algorithm}

Within the merge phase, pertinent leaves are reduced pairwise in any given order. This includes the pairwise reduction of pertinent leaves of different components as well. Hence, components that have pertinent leaves of the same vertex in their frontier, are merged in an arbitrary order.

Consider four different components $F_{1}, F_{2}, F_{3}, F_{4}$ and their corresponding $P Q$-trees $T_{1}, T_{2}, T_{3}, T_{4}$ each having at least one pertinent leaf corresponding to some level- $j$ vertex $k$. For simplicity, assume that the pertinent leaves of every component appear consecutively in all permutations on one side of their $P Q$-trees and assume further that the smallest common ancestor of the pertinent leaves and some other leaves is a $Q$-node. In Fig. 6 such a component $F_{i}, i \in\{1,2,3,4\}$, and its corresponding $P Q$-tree $T_{i}, i \in\{1,2,3,4\}$, is shown. The number $c_{i}$, $i \in\{1,2,3,4\}$, depicts the ML-value between the leftmost pertinent leaf and the frontier of its left neighbor. We have marked all pertinent leaves with a $k$ for simplicity.

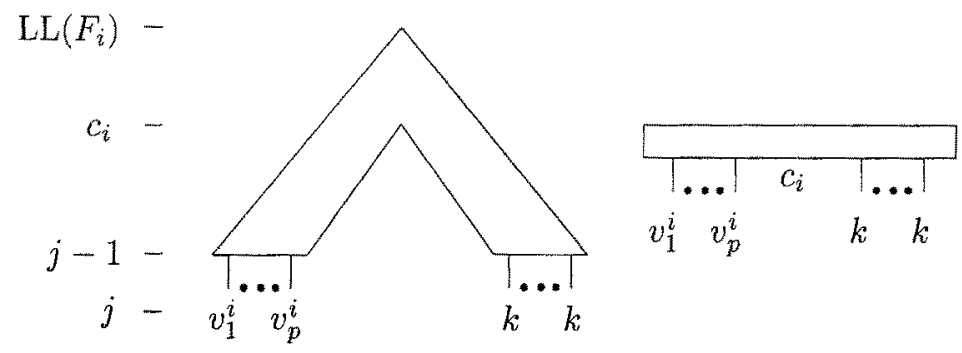

Fig. 6. Component $F_{i}$ and its corresponding $P Q$-tree $T_{i}$. On the left side of $F_{i}$, some levels of $F_{i}$ are indicated. The value $c_{i}$ is equal to $\mathrm{ML}\left(\left\{v_{p}^{i}, k\right\}\right)$.

Assuming that the following condition,

$$
L L\left(F_{1}\right) \leq c_{1}<L L\left(F_{2}\right) \leq c_{2}<L L\left(F_{3}\right) \leq c_{3}<L L\left(F_{4}\right) \leq c_{4}
$$

on the ML- and LL-values of the components holds, it is possible to merge all four components into one component such that the pertinent leaves form a consecutive sequence. Figure 7 shows the four components, indicating how the components can be merged so that a reduction of the pertinent leaves becomes possible.

Consider the following merge operations on the components $F_{1}, F_{2}, F_{3}, F_{4}$ and their corresponding $P Q$-trees:

1. merge $F_{1}$ and $F_{4}$ into component $F^{\prime}$,

2. merge $F^{\prime}$ and $F_{3}$ into component $F^{\prime \prime}$,

3. merge $F^{\prime \prime}$ and $F_{2}$ into component $F^{\prime \prime \prime}$. 


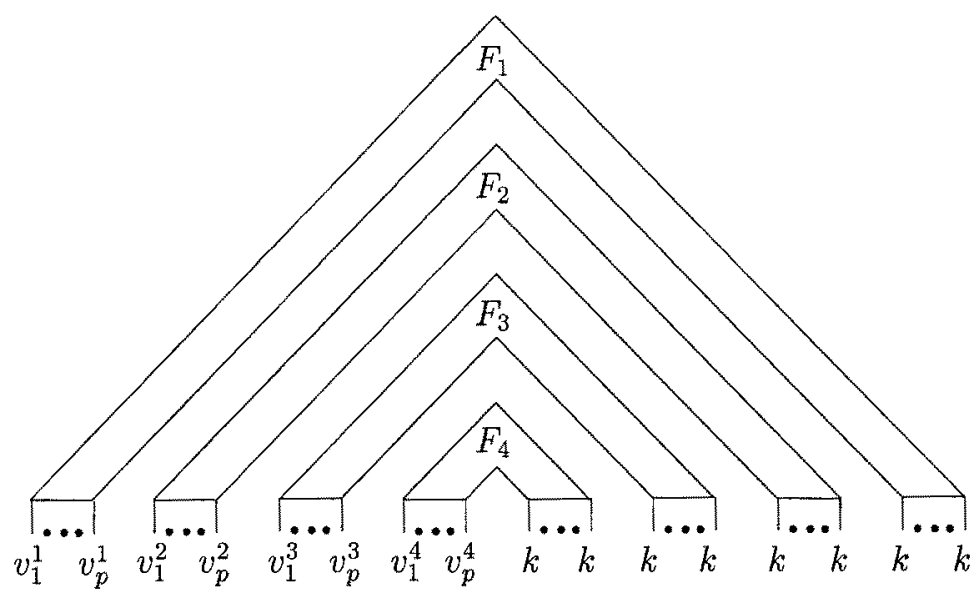

Fig. 7. Possible leveled planar arrangement of the components $F_{1}, F_{2}, F_{3}, F_{4}$.

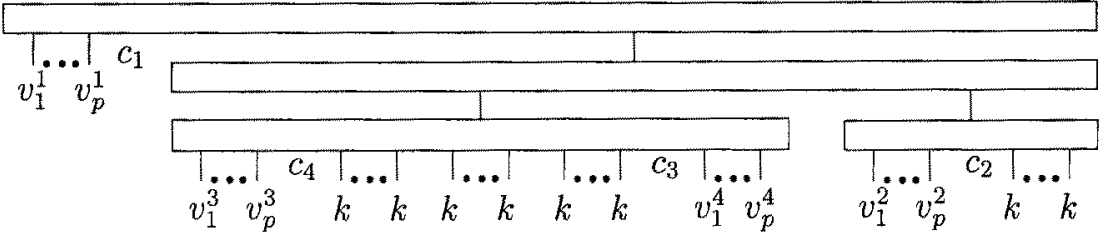

Fig. 8. $P Q$-tree $T^{\prime \prime \prime}$ whose pertinent leaves depicted by $k$ are not reducible.

The resulting $P Q$-tree $T^{\prime \prime \prime}$ corresponding to $F^{\prime \prime \prime}$ is shown in Fig. 8. Obviously, the pertinent leaves do not form a consecutive sequence in any permissible permutation of the $P Q$-tree. Hence the algorithm presented by Heath and Pemmaraju $(1996 \mathrm{a}, 1996 \mathrm{~b})$ states leveled nonplanarity although the graph may be leveled planar.

As a matter of fact, the order of merging the components is important for testing a leveled dag. Moreover it is easy to see, that using different orderings while merging three or more components results in different equivalence classes of $P Q$-trees. So even if every order of merging $P Q$-trees with pertinent leaves results in a reducible $P Q$-tree, a $P Q$-tree may be constructed such that the leaves of some vertex $l, \operatorname{lev}(l)>j$ are not reducible, although the graph $G$ is leveled planar. Hence the algorithm presented by Heath and Pemmaraju (1996a, 1996b) may state incorrectly the leveled nonplanarity of a leveled planar graph.

\section{References}

Booth, K. and Lueker, G. (1976). Testing for the consecutive ones property, interval graphs, and graph planarity using PQ-tree algorithms. Journal of Computer and System Sciences, 13, 335-379.

Chiba, N., Nishizeki, T., Abe, S., and Ozawa, T. (1985). A linear algorithm for embedding planar graphs using PQ-trees. Journal of Computer and System Sciences, $30,54-76$. 
Di Battista, G. and Nardelli, E. (1988). Hierarchies and planarity theory. IEEE Transactions on systems, man, and cybernetics, 18(6), 1035-1046.

Even, S. (1979). Graph Algorithms. Computer Science Press, Potomac, Maryland.

Even, S. and Tarjan, R. E. (1976). Computing an st-numbering. Theoretical Computer Science, 2, 339-344.

Heath, L. and Pemmaraju, S. (1996a). Recognizing leveled-planar dags in linear time. In F. J. Brandenburg; editor, Proc. Graph Drowing '95, volume 1027 of Lecture Notes in Computer Science, pages 300-311. Springer Verlag.

Heath, L. and Pemmaraju, S. (1996b). Stack and queue layouts of directed acyclic graphs: Part II. Technical report, Department of Computer Science, Virginia Polytechnic Institute \& State University.

Jayakumar, R., Thulasiraman, K., and Swamy, M. (1986). On maximal planarization of non-planar graphs. IEEE Transactions on Circuits Systems, 33(8), 843-844.

Jayakumar, R., Thulasiraman, K., and Swamy, M. (1989). On $O\left(n^{2}\right)$ algorithms for graph planarization. IEEE Transactions on Computer-Aided Design, 8(3), 257267.

Jünger, M., Leipert, S., and Mutzel, P. (1996). On computing a maximal planar subgraph using $\mathrm{PQ}$-trees. Technical Report 96.227, Institut für Informatik der Universität zu Köln.

Kant, G. (1992). An $O\left(n^{2}\right)$ maximal planarization algorithm based on PQ-trees. Technical Report RUU-CS-92-03, Department of Computer Science, Utrecht University.

Lempel, A., Even, S., and Cederbaum, I. (1967). An algorithm for planarity testing of graphs. In Theory of Graphs: International Symposium: Rome, July 1966, pages 215-232. Gordon and Breach, New York.

Ozawa, T. and Takahashi, H. (1981). A graph-planarization algorithm and its application to random graphs. In Graph Theory and Algorithms, volume 108 of Lecture Notes in Computer Science, pages 95-107. Springer Verlag. 